\title{
The influence of osteopathic treatment on pregnant women on the expected date of delivery on the course of parturition and the subjective well-being: A Pilot Study
}

Andrea Tauch $\mathrm{MSc}^{1}$

\section{ABSTRACT}

Background: The reduction of birth complications is of high interest in terms of mother and child's physical and mental health. Furthermore, passing the calculated birth date comes along with physical and psychological stress in many late-term pregnant women.

Objective: The purpose of this study was to explore the effect of one osteopathic treatment on selected birth complications and on the subjective well-being of the mother. The impact of OMT on vital signs and the actual date of delivery and the need for labor inductions was observed. The women's subjective experience with the treatment was interrogated through a questionnaire.

Methods: 58 pregnant women took part in this pragmatic controlled pilot study, including one treatment group ( $n=29)$ and one control group with usual obstetric care $(n=29)$. The treatment group received one osteopathic treatment on the expected date of delivery. Spielberger's State test, fetal heart rate, mother's heart rate and blood pressure were compared before and after the treatment tested through t-test. Selected maternal complications (using the Fisher Exact Test), actual delivery date, and drug-induced labor were compared between groups.

Results: No differences in the rate of selected complications were observed between groups $(p=1)$. The subjective well-being showed significant differences before and after treatment $(p<0.001)$ and correlated with the questionnaire analysis. The birth date was significantly earlier in the treatment group than the control group $(p=0.002)$. No statistically significant differences were observed in terms of drug-induced labor induction $(p=0.104)$.

Conclusions: In terms of birth complications, further studies with a higher number of treatments, earlier onset of therapy and treatment during birth with randomized subjects and three groups are highly recommended. Significant effects on the subjective well-being and the due date could be observed which indicate further studies.

Keywords: pregnancy, birth process, osteopathic treatment, subjective well-being

DOI: https://doi.org/10.35740/2020.2.1.2 


\section{BACKGROUND}

There is a long tradition in treating pregnant women through osteopathic medicine, not only in pregnancy-related symptoms but also in terms of delivery conditions. Already in 1911 and 1918, Whiting and Hart reported a reduced duration of labor and fewer deliveries using forceps in women who received prenatal OMT. [1]

Still, there is a lack of meaningful studies exploring the effect of osteopathic treatments on labor and delivery. As it is of highest interest to reduce birth-related complications to support the physical and mental health of mother and child, it is important to search for best ways to approach and promote it from the osteopathic point of view. The randomized controlled study of Keurentjes showed that OMT during the birth process could significantly reduce maternal morbidity in terms of rate of cesarean sections, episiotomies and perineal lacerations in the treatment group of 33 compared with a control group of 32 parturient women. [2] Also, the results of King et al.'s retrospective case-control design study support the hypothesis that prenatal OMT reduces the occurrence of some complications of labor and delivery and that as few as one or two OMT visits appeared to have a benefit. [1] In terms of safety, Hensel et al. showed that there is no increased risk of precipitous labor, operative vaginal delivery, conversion to cesarean delivery, need for forceps or vacuum device, need for episiotomy, incidence of perineal laceration, or meconium-stained amniotic fluid in the treatment group (7 scheduled treatment visits from 30 weeks of gestation on) when compared with participants in the other 2 groups (usual care plus placebo ultrasound treatment and usual care only). [3]

Still, there is a lack of well-designed studies that approach the late-term pregnant woman's treatment in a holistic osteopathic way, including all possible systems with Open-Box-Approach reporting details on the techniques used.
Furthermore, many pregnant women are physically and psychologically burdened as the expected date of delivery approaches and even more stressed if the calculated date of birth passes by. Many expectant mothers describe the fear of childbirth and general discomfort in late-term pregnancy. [4] To approach this matter of fact, in this study, the effect of OMT on the subjective well-being of the expectant mother has observed and the influence of osteopathic treatment regarding the onset of the birth process. As there is a lack of significant studies that describe the possibility of influencing the start of the birth process by OMT in the late-term pregnancy, this is of high interest. [5], [1]

Of an organizational point of view and as a matter of fact that OMT during delivery is not usual in Austria, it is easier to treat the late-term pregnant women as part of a clinical medical inspection on the calculated date of birth. This also supplies objective data of an accurately heterogeneous group.

This study investigates if maternal complications (secondary cesarean sections, forceps and vacuum-assisted deliveries, episiotomies and perineal lacerations degree III and IV) can be reduced by one osteopathic treatment using the OPEN-BoxApproach on the expected date of delivery. A further objective was to evaluate the effect of the osteopathic treatment on the subjective well-being of the late-term pregnant women using the State Inventory of Spielberger's State-Trait-AnxietyInventory. [6]

Furthermore, fetal heart rate, mother's heart rate and blood pressure were compared before and after the treatment. The actual date of birth following the calculated date of birth (and the treatment) and the number of drug-induced labor induction was observed and matched between the treatment and usual care groups.

After delivery, the osteopathic treatment women were asked about their subjective experience with it through a questionnaire. 


\section{METHODS}

\section{Design overview}

This study was designed as a pragmatic controlled clinical pilot study.

\section{Subjects/Recruitment}

A total of 59 pregnant women at the calculated date of delivery were recruited for this study. They were approached about this study during a regularly scheduled obstetrical visit at the hospital at 36 weeks of gestation and provided information about the study. Suppose the participant reached the calculated date of delivery and written informed consent was obtained as well as inclusion and exclusion criteria were observed. In that case, the woman could participate in the study.

The first 30 volunteers made up the treatment group. After the medical examination (measurements of maternal heart rate, maternal blood pressure, recording of uterine contractions and fetal heart rate, ultrasound, urinalysis), the treatment group members were given an osteopathic treatment after completing the State Test of the Spielberger's STAI. 15 Minutes after the treatment, the participants, once again, were given the State Test and vital signs and CTG check-up. After that, the women were sent home, and objective data about the birth process was noted at the actual date of delivery. The women were given a questionnaire before they went home from hospital with their child to evaluate the subjective experience with the osteopathic treatment.

One woman of the treatment group was excluded because of the decision for primary c-section before the onset of the birth process, so 29 women of the treatment group remained.

The next 29 women acted as the control group. After the standard medical examination, including a CTG, the group participants were asked to fill out the State-Test, but no osteopathic treatment was administered. After that, the women were sent home.

Participants could withdraw at any time of the study, and refusal to participate did not influence their further obstetrical care.

\section{Inclusion and Exclusion Criteria}

To enable enough women to volunteer in this study in the planned time period there were no restrictions with regard on age, race, and number of previous births. Also, women after a previous cesarean section were eligible for inclusion.

Participants were ineligible for inclusion if they expected multiple births, if the baby was in breech presentation and if a primary c-section was planned. Also, pregnant women who were deemed high risk by the obstetrical provider could not take part in this study.

Participants who were either below or over the calculated date of birth plus/minus one day $(39+6$ to $40+1)$ were excluded from the study. Expectant mothers, who gave their consent to the study but asked for primary c-section during parturition, were excluded.

Participants who already had received osteopathic treatment in the current pregnancy were not allowed to participate in this study.

\section{Treatments}

Each OMT group participant received a 45-minute osteopathic open-box treatment after findings. Techniques used included soft tissue, articulatory, HVLA just for the thoracic spine and only for very restricted blockades, myofascial release, muscle energy treatment, balanced ligamentous/membranous / fluid tension, visceral and cranial techniques. Also, a biodynamic approach was in use.

\section{STATISTICS}

For collecting data, the CTG and patient data sheet provided by the hospital were used. Baseline data were compared across treatment groups with a two-way analysis of variance. An alpha level of 0.05 was set for significance. 


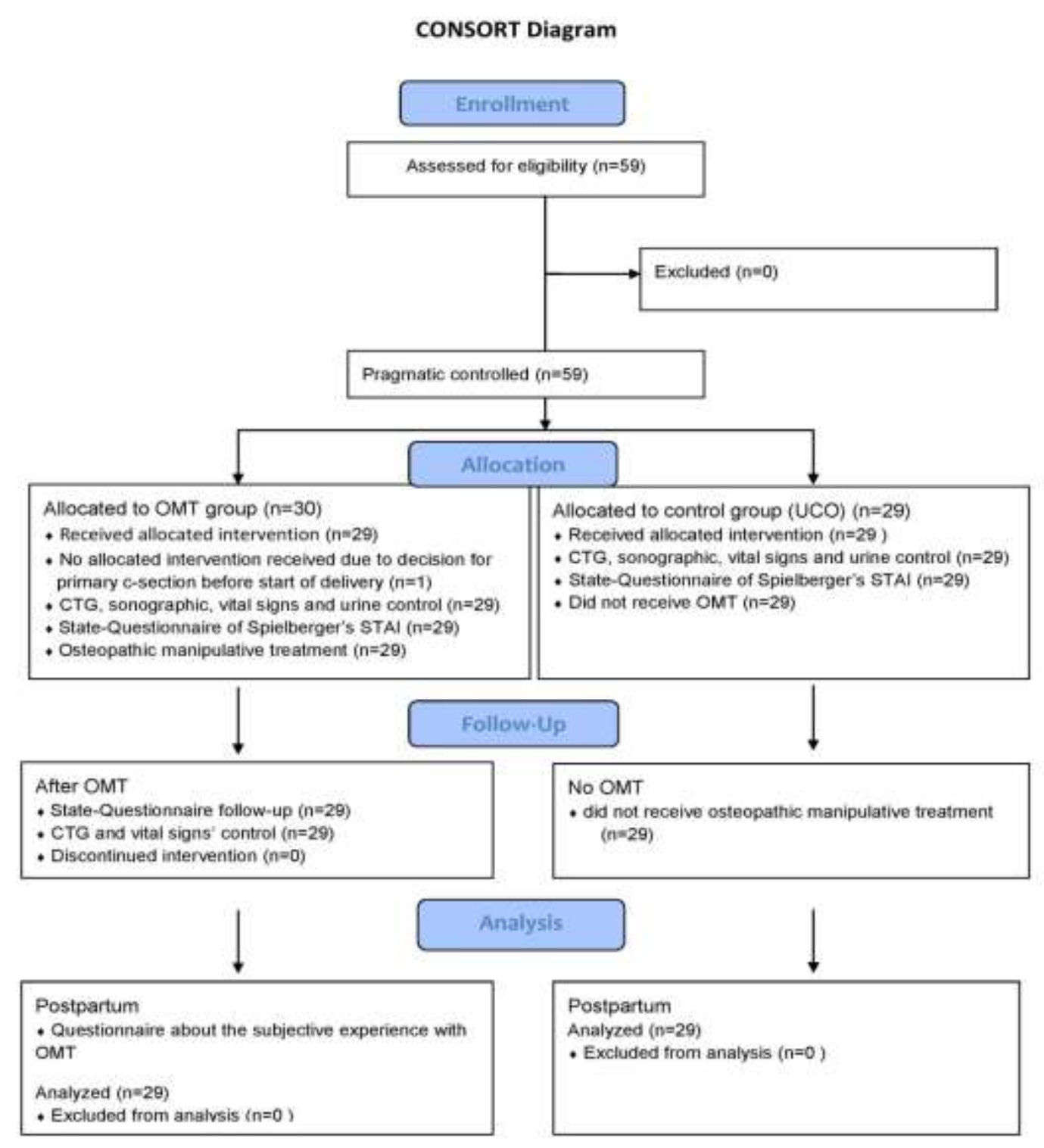

Figure 1: Participant flow

\section{Research questions:}

Research question 1: Can an Osteopathic Treatment on the calculated date of delivery $+/$ - one day reduces the risk of maternal complications (secondary cesarean section, vacuum assisted delivery, forceps assisted delivery, episiotomy, perineal laceration degree III and IV) in comparison to data of non treated women?
The Fisher Exact Test for both groups was not significant.

Research question 2: Can an Osteopathic Treatment on the calculated date of delivery $+/$ - one day improve subjective well-being according to State-Test of Spielberger's STAI.

The result of the paired t-test was $\mathrm{p}<0.001$. Which means there are significant differences 
Table 1: Demographic characteristics

\begin{tabular}{|l|c|c|}
\hline & Treatment group & Usual care onlygroup \\
\hline N & 29 & 29 \\
\hline Primigravida & 16 & 15 \\
\hline Multigravida & 13 & 14 \\
\hline Status post C-Section & 1 & 1 \\
\hline Median Age & 31 & 33 \\
\hline Mean Age & 29,31 & 30,9 \\
\hline
\end{tabular}

between the State-Score before and after the osteopathic treatment. So Research question 2 can positive answered.

A further parameter that was proved before and 15 Minutes after the treatment were the maternal blood pressure and heart rate and the fetal heart rate using the dependent $\mathrm{t}$-test.

Also, the difference between the actual delivery date and the calculated date of delivery was compared between treatment and control groups and statistically evaluated by $\mathrm{t}$-test for independent samples.

The quantity of drug-induced labor inductions was analyzed by Fisher exact test.

\section{RESULTS}

Complete data sets were obtained in 58 subjects (29 OMT group, 29 control group). The loss of one participant from the total 30 in the treatment group was due to the wish for a primary c-section of one woman. To have the same quantity of subjects in both groups, only 29 participants were enrolled for the control group.

There were no significant differences in baseline blood pressure, maternal and fetal heart rate and the State Test between groups. CONSORT diagram showing subject flow through the study is Figure 1.

\section{Maternal complications}

Maternal complications included were secondary c-section, vacuum-assisted delivery, forceps assisted delivery, episiotomy, perineal laceration degree III and IV.

No differences in the quantity of occurred defined complications were observed between the treatment and control group (usual care only) $(\mathrm{p}=1)$ as listed in table 1 below.

\section{Subjective well-being / State-Score}

A highly significant decrease in the State score of Spielberger's STAI was observed $(p<0.001)$ pre to post-treatment. The decrease of the State score averaged at 11.03 points.

\section{Maternal blood-pressure}

The systolic and diastolic blood pressure was observed pre to post-treatment. Both decreased significantly before to 15 minutes after the treatment ( $\mathrm{p}$-value for the systolic blood-pressure: $\mathrm{p}=0.008$, diastolic blood-pressure: $\mathrm{p}=0.004$ ).

\section{Maternal heart rate}

On average, the maternal heart rate decreased about 7 heartbeats per minute pre to 15 minutes post treatment. The statistic's result revealed a $\mathrm{p}$ $<0.001$, therefore a highly significant decline of the heart rate could be noticed.

\section{Fetal heart rate}

The fetal heart rate also decreased highly significant before to 15 minutes after the treatment with a confidence level lying between 2.289 and 8.746 $(\mathrm{p}=0.002)$. 
Table 2: Type of birth / Complications

\begin{tabular}{|c|c|c|}
\hline $\begin{array}{l}\text { Type of birth/Complications: } \\
\text { (by grouping) }\end{array}$ & Treatment group & Control group \\
\hline Induced & 4 & 9 \\
\hline Secondary caesarean section & 7 & 7 \\
\hline Vacuum extraction & 3 & 2 \\
\hline Eplsiotomy & $\begin{array}{c}2 \\
\text { (with vacuum extraction) }\end{array}$ & (with vacuum extraction) \\
\hline Perineal laceration 3rd degree & (with vacuum extraction) & 0 \\
\hline Perineal laceration 4th degree & 0 & 1 \\
\hline $\begin{array}{l}\text { Spontaneous deliveries } \\
\text { (without complications and inductions) }\end{array}$ & 12 & 8 \\
\hline
\end{tabular}

\section{Actual date of delivery after expected date of delivery}

The onset of parturition after due date was earlier in the treatment group than the control group. In average, participants of the treatment group had their baby 4.55 days after the due date (and the treatment), women of the control group 7.48 days after the expected date of delivery (EDD).

Statistics show $\mathrm{p}=0.002$, so the result is significant.

Table 3 summarizes the frequencies of participant going beyond the EDD. EDD+0, therefore, is the expected/calculated date of birth, EDD +3 for example, means the actual date of birth was three days after the calculated date of delivery. Table 2 shows that only five participants $(17.2 \%)$ of the treatment group gave birth after one week after EDD, that means in the 43rd week of pregnancy. In the control group, 14 women (48.3\%) gave birth to their babies not until the 43rd week. In the first three days after EDD and therefore also after the osteopathic treatment, 11 women $(37.9 \%)$ of the treatment group delivered their babies compared to three women $(10.3 \%)$ in the control group.

\section{Drug-induced labor inductions}

There is no significant influence of the treatment on drug-induced labor inductions. The Fisher exact test was performed with a confidence level of $95 \%$ and showed a p-rating of 0.104 . In absolute figures there were 9 inductions in the control group and 4 in the treatment group.

\section{Subjective experience with OMT / Ques- tionnaire after delivery}

The questionnaire after delivery revealed that $89.7 \%$ (26 of 29 participants) would very much (22 women) or rather like ( 4 women) to have OMT when having a further baby.

$89.7 \%$ had also observed positive changes in their physical condition after OMT. In terms of emotional/psychological state, 16 women $(55.2$ $\%)$ had felt better after OMT. 21 women (72.4\%) reported better sleep after the treatment. 58.6\% of the study's participants had the impression that the OMT positively had influenced the onset of labor, 19 women $(65.5 \%)$ had the feeling that the OMT had positively affected the course of birth. All the study participants felt comfortable during the treatment, and 28 out of 29 women stated 
Table 3: Actual date of delivery compared to expected date of delivery (EDD+)

\begin{tabular}{|l|l|l|}
\hline EDD & Treatment group & Control group \\
\hline$E D D+0$ & 2 & 1 \\
\hline$E D D+1$ & 1 & 1 \\
\hline$E D D+2$ & 8 & 1 \\
\hline$E D D+3$ & 1 & 3 \\
\hline$E D D+4$ & 5 & 3 \\
\hline$E D D+5$ & 2 & 1 \\
\hline$E D D+6$ & 2 & 2 \\
\hline$E D D+7$ & 3 & 3 \\
\hline$E D D+8$ & 3 & 1 \\
\hline$E D D+9$ & 0 & 3 \\
\hline$E D D+10$ & 2 & 3 \\
\hline$E D D+11$ & 0 & 5 \\
\hline$E D D+12$ & 0 & 1 \\
\hline$E D D+13$ & 0 & 0 \\
\hline$E D+14$ & 0 & 1 \\
\hline
\end{tabular}

that the treatment had relaxed them. So in terms of the subjective well-being, OMT seems to provide positive support.

\section{DISCUSSION}

This study addresses the effects of osteopathic manipulative treatment on maternal complications, the subjective well-being of the late term pregnant women, the autonomic nervous system, and the onset of delivery. To the author's knowledge it is the first study to observe the effects on the onset of birth with regard on a clinical osteopathic approach on specific diagnoses and not only the CV4 for example. [1], [7]

The primary finding was that one OMT on the expected delivery date could improve the subjective well-being according to the State Test of Spielberger's STAI of the expectant mother pre to post-treatment. It seemed that the treatment had a calmative effect on the autonomic nervous system. It can be hypothesized that this may affect the delivery's significant earlier onset after the treatment group's due date. As there were no differences in maternal complications, it seems that one treatment without supporting the delivery with OMT has too less influence and/or other effects are overruling.

\section{Discussion of methods/study design}

The study design with one treatment group and one control group (usual care only) reveals first impressions. To evaluate the specific osteopathic effect, it would be necessary to bring in a 3rd group - for example, a conversation or massage group.

There was no randomization carried out for this study which had organizational reasons. The control group was run after the treatment group had finished ensuring a sample as homogenous as possible. A higher sample group and randomized control are highly recommended for further studies. Furthermore, it is absolutely necessary to measure State-Score, maternal blood pressure 
and heart rate, and fetal heart rate of the control group a second time (for example, time control). There were no restrictions with regard on age, race, and number of previous births to ensure enough volunteers for the study. Also, women after a previous cesarean section were eligible for inclusion. In further studies it is recommended to include for example only nulliparous women or only multiparous women. As there is a high probability for another cesarean section after having already one, the exclusion of women with previous cesarean sections could also be considered. The cesarean section rate is higher in women with a high Body-Mass-Index; therefore, it can be discussed to exclude obese pregnant women in further studies. Other alternative methods (for example, acupuncture, herbal medicine, and perineal massage) were not prohibited in this study to ensure enough participants. Also, many of these methods were ongoing anyway at the first contact in the hospital.

For the evaluation of the subjective well-being, only the State Test of STAI was used. It could be considered to take the whole State-Trait-AnxietyInventory or the Wijma Delivery Expectancy Scale (W-DEQ) to compare other studies in this field well as to get more exact data.

\section{Effects on maternal complications}

There was no influence of one osteopathic treatment on the expected delivery date on the rate of secondary cesarean sections, vacuum deliveries, episiotomies and perineal lacerations degree III and IV. It must be considered that the birth of a child is a very dynamic process which is dependent on many influencing factors, for example, the preparation for delivery, the attitude of the woman towards birth, support of the accompanying person, birth management and experience of the midwife/obstetrician, position of birth, anatomic conditions (for example cephalopelvic disproportion, nuchal cord) and furthermore.

It might be assumed that all prevailing factors eventually decide how the birth process proceeds. It is recommended to probably reduce birth complications to have a higher frequency of treatments, begin earlier in pregnancy with the treatments, and support the women through delivery with OMT. Studies have already evaluated this. [3], [8], [1], [2]

Also, with a higher frequency of treatment, there would be a better mutual of trust to carry out vaginal and/or rectal techniques if needed, which could be a significant factor in reducing complications because of the better possibility of preparing the perineal structures.

Moreover, it is suggested to relate maternal complications to the birth weight and the child's head circumference in further studies.

\section{Effects on subjective well-being / State- Score}

Evaluation of statistics showed that the StateScore is highly significantly decreasing pre to post-treatment $(\mathrm{p}<0.001)$. It could be hypothesized that the OMT can reduce the nervous system's tension to stimulate the beginning of the birth process. As the actual delivery date is statistically significant earlier in the treatment group than the control group, this can cautiously be assumed in this study.

Some factors must be discussed critically. Firstly, there was no comparison of the measurements to a control group (except the baseline data, which was homogenous). Due to organizational factors and the very probable loss of participants in the control group, there was no second evaluation of vital sign's data or the State-Score in the control group after passing the time. To identify the specific osteopathic effect, it is necessary to evaluate the OMT data compared to time control and placebo group or other groups. Furthermore this study does not address the duration of the reduction of the State-Score, so further research is needed to evaluate the potential benefit of OMT.

\section{Effects on maternal blood pressure}

The systolic, as well as the diastolic blood pressure, decreased significantly pre to 15 minutes post-treatment ( $p$ syst. $=0.008, p$ diast. $=0.004)$. This must be observed critically as there is no comparison to a control group's time control measurement. Also, it must be discussed if the reduction of the blood pressure is a desirable effect in every pregnant woman; this did not issue this 
study, but it must be mentioned that no woman had subjective negative effects of her circulatory system after the treatment. In order to prove the effect of OMT to hemodynamic control, a tilt stimulus and/or heel raise effect could be added in further studies. [9]

\section{Effects on maternal heart rate}

Maternal heart rate declined seven beats per minute on average compared pre to 15 minutes post-treatment $(\mathrm{p}<0.001)$. As mentioned before, this must be observed and compared at least to a time control group in further studies.

\section{Effects on fetal heart rate}

Fetal heart rate decreased 5.5 beats per minute on average in comparison pre to 15 minutes after the treatment $(\mathrm{p}=0.002)$. This effect has to be evaluated in further studies by taking at least a time control group into account.

\section{Effects on the actual date of delivery}

Statistics revealed a highly significant effect of OMT $(p=0.002)$ in terms of the actual birth after due date compared between treatment to control group. As mentioned before one could hypothesize that the OMT can reduce tension of the nervous system (also confirmed by the State-Score) and therefore possibly influence the beginning of the delivery course. This would have high medical relevance, as post-term pregnancies can be dangerous in terms of the child's undersupply. If labor does not begin appropriately, drug-induced labor induction follows, which, in turn, leads more often to complications.

\section{Effects on the need for labor inductions}

There were nine inductions in the control group, and 4 in the treatment group $(\mathrm{p}=0.104)$. With a bigger random sample in further studies, this tendency might produce significant results. It would be recommended in further studies to record the reason for the induction to see if it was medically necessary or the mother's personal request. From a medical point of view, the reduction of druginduced labor inductions is to be strived as they often lead to uterine over-stimulation, which frequently results in fetal distress and early exhaustion of the mother. Induced labor is often reported as being more painful and often leads to necessary pain-relieving interventions. Primiparas have a higher probability of cesarean sections after drug-induced labor inductions than women with natural onset of labor. [10]

\section{CONCLUSIONS}

This study demonstrated that one osteopathic treatment on the calculated date of delivery was not sufficient enough to reduce maternal complications, but did show a better physical and psychological well-being of the mothers-to-be after the Open-Box treatment. This and the possible influence of the autonomic system might have led to significant earlier deliveries in the OMT group compared to the control group (UCO) which is of high medical interest to prevent the need for labor inductions. Data showed that maternal blood pressure and heart rate and fetal heart rate, declined after the treatment - this has to be further investigated in more appropriate methodological design. The possibility of reducing overall sympathetic tone with OMT and its incidence of associated potential complications of pregnancyrelated autonomic and hemodynamic conditions (for example pregnancy-induced hypertension and preeclampsia) is of great importance to healthcare and needs further studies. [9]

In conclusion, this study confirms the positive effect of OMT on the subjective well-being of the late-term pregnant woman and the onset of delivery - also with only one treatment. Moreover, it reveals the cautious conclusion that OMT could affect the autonomic system. To prevent maternal complications, a higher frequency of treatments and/or an Osteopath's support during delivery seems to be necessary.

\section{Discloser}


The author has no personal, financial or institutional interest in any of the drugs, materials or devices described in this article.

\section{Author details}

${ }^{1}$ A.ö. Krankenhaus Spittal/Drau

\section{Correspondence}

Andrea Tauch

Schlossau 48

9871 Seeboden

Austria

Email: andrea.tauch@gmx.at

Received: 23 August 2020 Accepted: 13 December 2020 Published: 20 December 2020

\section{REFERENCES}

1. H. H. King, M. A. Tettambel, M. D. Lockwood, K. H. Johnson, D. A. Arsenault, and R. Quist, "Osteopathic manipulative treatment in prenatal care: a retrospective case control design study," J. Am. Osteopath. Assoc., vol. 103, no. 12, pp. 577-582, Dec. 2003.

2. E. Keurentjes, "Relationship of Osteopathic Manipulative Treatment during Labor and Delivery on Selected Maternal Morbidity Outcomes: A Randomized Controlled Trial.," Doctor of Philosophy, Faculty of the Virginia Polytechnic institute and State University, Blacksburg, 2009.

3. Hensel, K. L., Roane, B. M., Chaphekar, A. V., \& Smith-Barbaro, P. (2016). PROMOTE Study: Safety of Osteopathic Manipulative Treatment During the Third Trimester by Labor and Delivery Outcomes. The Journal of the American Osteopathic Association, 116(11), 698.

https://doi.org/10.7556/jaoa.2016.140

4. Westfall, R. E., \& Benoit, C. (2004). The rhetoric of "natural" in natural childbirth: childbearing women's perspectives on prolonged pregnancy and induction of labour. Social Science \& Medicine, 59(7), 1397-1408.

https://doi.org/10.1016/j.socscimed.2004.01.017

5. T. Liem, Kraniosakrale Osteopathie. Ein praktisches Lehrbuch., 5th ed. Stuttgart: Hippokrates Verlag, 2010.
6. J. Grimm, "State-Trait-Anxiety Inventory nach Spielberger. Deutsche Lang- und Kurzversion.- Methodenforum der Universität Wien.," 2009. [Online]. Available: https://empcom.univie.ac.at/fileadmin/user upload/p empcom/Grimm2009_StateTraitAngst_MFWorkPaper2009-02.pdf. [Accessed: 29-Sep-2018].

7. D. Samonigg-Lackner, "Einfluss der cranioacralen Behandlung CV-4 bei Schwangeren in der 41. Schwangerschaftswoche," Masterthese, DonauUniversität Krems, Pesenthein, 2017.

8. Licciardone, J. C., Buchanan, S., Hensel, K. L., King, H. H., Fulda, K. G., \& Stoll, S. T. (2010). Osteopathic manipulative treatment of back pain and related symptoms during pregnancy: a randomized controlled trial. American Journal of Obstetrics and Gynecology, 202(1), 43.e1-43.e8.

https://doi.org/10.1016/j.ajog.2009.07.057

9. Hensel, K. L., Pacchia, C. F., \& Smith, M. L. (2013). Acute improvement in hemodynamic control after osteopathic manipulative treatment in the third trimester of pregnancy. Complementary Therapies in Medicine, 21(6), 618-626. https://doi.org/10.1016/j.ctim.2013.08.008

10. S. Sedlak, "Probleme und Gefahren der künstlichen Geburtseinleitung - PDF," 2006. [Online]. Available: https://docplayer.org/15763092-Probleme-und-gefahren-der-kuenstlichen-geburtseinleitung.html. [Accessed: 11-Jul-2019]. 